\title{
Alternative of Shortage Lorazepam in Case of Status Epilepticus
}

\section{Abdulrahman Hadi Almutiri*}

25 abi alhareth street, Orijha, Riyadh, P.O. Box 315965, Riyadh 11331, Saudi Arabia

*Corresponding author: Abdulrahman Hadi Almutiri, 25 abi alhareth street, Orijha, Riyadh, P.O.Box 315965, Riyadh 11331, Saudi Arabia, Tel: 966553228474; E-mail: abrhp2006@hotmail.com

Received date: November 21, 2015; Accepted date: November 27, 2015; Published date: December 03, 2015

Copyright: ( 2015 Almutiri $\mathrm{AH}$. This is an open-access article distributed under the terms of the Creative Commons Attribution License, which permits unrestricted use, distribution, and reproduction in any medium, provided the original author and source are credited.

\section{Short Communication}

A suitable alternative to lorazepam in status epilepticus (SE) is understandable given the shortage of the injection formulation arising from manufacturing problems. I have consulted certain professional literature relating to this matter, including Section 4.8.2 of the British National Formulary (BNF, 2011) which describes drugs used in SE and the recently update guidelines from the National Institute for Clinical Excellence (NICE) which describe treatment of epilepsy in adults and children in primary and secondary care (NICE, 2012). This latter publication has been augmented with an online flowchart with a section specifically relating to treating convulsive SE in adults and children [1]. This is an invaluable consultation tool, to which you may want to refer. Additionally, two Cochrane reviews investigating whether a particular anticonvulsant is more effective or safer than another in treating SE in adults and children [2,3] provide the most relevant, up-to-date research on drug therapy for SE.

Preventing seizure-associated morbidity and mortality requires effective and fast-acting drugs. Benzodiazepines (BZ) fulfil these criteria, making them ideal first choice anti-epileptic drugs (AED) for initial treatment of SE. To achieve rapid attainment of therapeutic levels in SE, the ideal route for administration is intravenous (IV), providing of course facilities for resuscitation are available due to the potential for respiratory depression or hypotension. IV lorazepam is the first-line therapy for SE (BNF, 2011). In the absence of UK licensed lorazepam injection, imported supplies of the drug may be available, such as from the USA, but these unlicensed, expensive alternatives may be unacceptable. Therefore, during the shortage, alternative injectable BZ, specifically diazepam and midazolam, should be considered. Lorazepam injection superseded IV diazepam as first choice for treating SE largely due to its smaller volume of distribution hence longer duration of action, the more lipid soluble diazepam being readily redistributed away from the brain to other tissues (Table 1). Many clinical trials have actually shown no difference in seizure control between these drugs (see Cochrane reviews) and although the BNF (2011, p298) recommends IV lorazepam as first-line treatment, it does state that diazepam is also effective, recommending use of its emulsion formulation to overcome thrombophlebitis problems. Midazolam has a marginally longer duration of action compared with diazepam (Table 1) but it is less accessible since it must be prescribed and ordered as a controlled drug. It is also more expensive than diazepam. However, in the absence of IV access, intramuscular (IM) midazolam is probably the fastest acting and easiest formulation to use (Table 1).

\begin{tabular}{|c|c|c|c|c|c|c|}
\hline \multirow[t]{2}{*}{ Agent } & \multicolumn{2}{|c|}{ Onset of Action (min) } & \multicolumn{2}{|c|}{ Duration of Action (hours) } & \multirow{2}{*}{$\begin{array}{l}\text { Half-life } \\
\text { (hours) }\end{array}$} & \multirow[t]{2}{*}{ Active Metabolites } \\
\hline & Intravenous & Intramuscular & Intravenous & Intramuscular & & \\
\hline Diazepam & $1-5$ & a & $0.3-0.5$ & a & $20-120$ & Yes \\
\hline Lorazepam & $5-20$ & $15-30$ & $6-8$ & $6-8$ & $8-15$ & No \\
\hline Midazolam & $1-5$ & $5-15$ & $\leq 2^{\mathrm{b}}$ & $2^{b}$ & 3-11 & Yes \\
\hline
\end{tabular}

Table 1: Pharmacokinetics of Injectable Benzodiazepines. ${ }^{a}$ Intramuscular administration results in slow and erratic absorption. ${ }^{\mathrm{b}}$ The pharmacologic effect of midazolam may last up to 6 hours in some patients.

Alternative therapies include those commonly recommended for the community setting namely rectal diazepam and midazolam given into the buccal cavity, this latter being an unlicensed use of midazolam, requiring written informed consent prior to administration.

Both of these formulations have a slower onset of action compared with the injectable formulations. Moreover, differences between midazolam and diazepam in terms of efficacy or side effects are minimal. Families thus have a choice of rescue medication used to manage prolonged seizures in the community. Many prefer the more socially acceptable buccal midazolam formulation, over rectal diazepam, especially for children. However, Buccolam, midazolam's pre-filled oral syringe formulation, is considerably more expensive than rectal diazepam. Midazolam has, however, shown to exhibit superior efficacy compared with diazepam in one paediatric study (see Cochrane report).

Individual patient factors impact on risks versus benefits of drug intervention, such as the risk of long-term neurological damage against potential for exacerbation of symptoms associated with an existing respiratory disease or risks to the foetus during pregnancy. Midazolam and diazepam have subtle differences in their safety profiles, specifically in patients with liver disease and in the elderly. Both drugs require dose reduction in hepatic and renal disease and in elderly patients a lower diazepam dose is given. However, due to diazepam's inherently long and variable elimination half-life $\left(t_{1 / 2}\right)$, which is directly related to its duration of sedation, comorbidities adversely affecting this variable are of particular significance. Diazepam $t_{1 / 2}$ in volunteers is around 1 day, whereas in severe liver disease it ranges 
Citation: Almutiri AH (2015) Alternative of Shortage Lorazepam in Case of Status Epilepticus. J Pharmacovigil 3: 186. doi: 10.4172/2329-6887.1000186

Page 2 of 2

from 75 to $150 \mathrm{~h}$ and in elderly volunteers it is 44 to $161 \mathrm{~h}[4,5]$. Midazolam on the other hand has a short $\mathrm{t}_{1 / 2}$ (around $4 \mathrm{~h}$ ), and even in severe alcoholic cirrhosis it only reaches on average $28 \mathrm{~h}$ [6]. Midazolam should thus be the drug of choice in hepatic disease and the elderly.

To conclude, where resuscitation facilities are available, and lorazepam injection is unavailable, diazepam should be the $\mathrm{BZ}$ of choice. Diazepam should be administered by slow IV injection, ideally using the emulsion formulation: $10 \mathrm{mg}$ : adults; $300-400 \mu \mathrm{g} / \mathrm{kg}$ : children under 12 years. If IV access cannot be obtained, it should be administered rectally: $10-20 \mathrm{mg}$ : adults; $10 \mathrm{mg}$ elderly; $5-10 \mathrm{mg}$ children 2-12 years (BNF for younger children) [7]. These doses can be repeated after 10 minutes if required. Also, co-administration of a long acting AED may be advisable. In the elderly, children or in those with hepatic disease, midazolam would be the preferred BZ. The choice of formulation, either IM injection or buccal solution would depend upon patient and clinician preferences, accessibility and cost.

\section{References}

1. http://pathways.nice.org.uk/pathways/epilepsy/

2. Prasad K, Krishnan PR, Al-Roomi K, Sequeira R (2007) Anticonvulsant therapy for status epilepticus. Br J Clin Pharmacol 63: 640-647.

3. Appleton R, Macleod S, Martland T (2008) Drug management for acute tonic-clonic convulsions including convulsive status epilepticus in children. Cochrane Database Syst Rev.

4. Divoll M, Greenblatt DJ, Ochs HR, Shader RI (1983) Absolute Bioavailability of Oral and Intramuscular Diazepam: Effects of Age and Sex. Anesth Analg 62: 1-8.

5. Barash PG, Cullen BF, Stoelting RK, Cahalan M, Stock C (2009) Clinical Anesthesia, Lippincott Williams and Wilkins, Philadelphia.

6. Macgilchrist AJ, Birnie GG, Cook A, Scobie G, Murray T, et al. (1986) Pharmacokinetics and pharmacodynamics of intravenous midazolam in patients with severe alcoholic cirrhosis. Gut 27: 190-195.

7. Epilepsies: diagnosis and management of the epilepsies in adults and children in primary and secondary care (2011) National Institute for Health and Clinical Excellence. 